Adamczyk Katarzyna, Kasztelan-Szczerbinska Beata, Rycyk Anna, Cichoz-Lach Halina. New view on alcoholic liver disease potential role of PD1/PDL-1 pathway in the disease pathogenesis. Journal of Education, Health and Sport. 2019;9(11):121-127. eISSN 2391-8306. DOI http://dx.doi.org/10.12775/JEHS.2019.09.11.009

https://apcz.umk.pl/czasopisma/index.php/JEHS/article/view/JEHS.2019.09.11.009

https://zenodo.org/record/3544837



\title{
New view on alcoholic liver disease - potential role of PD1/PDL-1 pathway in the disease pathogenesis
}

\author{
Katarzyna Adamczyk, Beata Kasztelan-Szczerbinska, Anna Rycyk, Halina Cichoz-Lach
}

Department of Gastroenterology with Endoscopy Unit, Medical University, Lublin

\section{Authors information and address for correspondence:}

1. Katarzyna Adamczyk MD, ORCID https://orcid.org/0000-0003-4861-7686, Department of Gastroenterology with Endoscopy Unit, Medical University, Lublin, e-mails: kasiaa890@wp.pl, kasiaa789@wp.pl, address for correspondence: Poland, Lublin 20-954, Jaczewskiego Street 8

2. Beata Kasztelan- Szczerbińska PhD, Assistant Professor, ORCID https://orcid.org/0000-0002-7198-4428, Department of Gastroenterology with Endoscopy Unit, Medical University, Lublin, e-mail: beata.szczerbinska@ op.pl

3. Anna Rycyk MD, ORCID https://orcid.org/0000-0001-5625-0330, Department of Gastroenterology with Endoscopy Unit, Medical University, Lublin, e-mail: aniarycyk@ op.pl 4. Halina Cichoz-Lach Professor, ORCID https://orcid.org/0000-0002-7337-835X, Department of Gastroenterology with Endoscopy Unit, Medical University, Lublin, email: halinacichozlach@umlub.pl

\begin{abstract}
Introduction: Exposure to chronic, excessive alcohol consumption, its breakdown metabolites, and gut-derived endotoxins dysregulate immune signaling leading to activation of inflammation. As a result, the non-resolving inflammatory response and development of alcoholic liver disease (ALD) may occur. There are still no pharmacological or nutritional therapies for treating patients with alcoholic liver disease.
\end{abstract}

Material and Methods: The information provided was collected as a result of various article's analyses on the development, diagnosis, and treatment of patients with the alcoholic liver disease using Google Scholar and PubMed databases.

Results: The pathway consisting of the programmed cell death 1 (PD-1) receptor and its ligand PD-L1 may play a critical role in the inhibition of self-reactive and inflammatory effector cells and the protection against immune-mediated tissue damage. There is a possibility of the potential gender-related differences in PD1/PD-L1 signaling in ALD. 
Conclusion: The better understanding of ALD as a dynamic process and all aspects of immune dysregulation may be important for the development of novel therapies in alcoholic liver disease. Results from a further study on the PD1/PDL-1 pathway might be exploited to re-define therapeutic targets and create sex-tailored treatments according to individual patient needs.

Keywords: alcohol liver disease (ALD); immune dysregulation; programmed cell death 1 (PD-1); gender difference

\section{Introduction and purpose of work}

Health problems resulting from excessive consumption of ethanol are relevant issue in most countries. Alcoholic liver disease (ALD) is a major cause of chronic liver disease worldwide and can lead to fibrosis and cirrhosis [5].

The latest surveillance report by WHO showed that worldwide in 2016, more than half ( $57 \%$ or 3.1 billion people) of the global population aged 15 years and over had abstained from drinking alcohol in the previous 12 months while about 2.3 billion people are current drinkers. Alcohol is consumed by more than half of the population in the Americas, Europe and Western Pacific. The prevalence of heavy episodic drinking (HED) - defined as 60 or more grams of pure alcohol on at least one occasion at least once per month - has decreased globally from $22.6 \%$ in 2000 to $18.2 \%$ in 2016 among the total population, but still remains high among drinkers, particularly in parts of Eastern Europe and in some sub-Saharan African countries (over 60\% of current drinkers). In 2016 the harmful use of alcohol was one of more frequent causes of 1.7 million deaths from noncommunicable diseases, including some 0.6 million deaths from digestive diseases [1]. The most common alcohol-attributable digestive diseases include liver cirrhosis and pancreatitis. Both of them are becoming more frequent cause of mortality and morbidity globally, mostly in developed countries [2], causing about 637000 digestive disease-related deaths in 2016. Alcohol-attributable pancreatitis resulted in 30000 deaths while alcohol-attributable liver cirrhosis caused almost 607000 deaths [1]. As it can be seen, heavy drinking can cause permanent damage to the liver mostly because it is the primary site of ethanol metabolism. Chronic and excessive alcohol consumption produces a wide spectrum of hepatic lesions, the most characteristic of which are steatosis, hepatitis, and fibrosis/cirrhosis [4].

Despite the ALD pathophysiology relates largely to the direct toxic effects of alcohol, the whole process of ALD development is complex and still incompletely understood. Till now there are no FDA-approved pharmacological therapies for treating this group of patients. The progress in understanding and new concepts of the ALD pathogenesis may create a chance to bring new ways of prevention and early intervention in order to slow the disease progression, avoid or delay its clinical decompensation or even the need for liver transplantation in the future.

\section{Hepatotoxicity of ethanol}

ALD is considered a complex disease in which numerous factors interact to allow for liver disease to occur. The cellular and molecular mechanisms of ALD pathogenesis are still incompletely understood but seem to be related to a complex interaction between behavioral, environmental and genetic factors [6]. A crucial component in the evolution of ALD is the direct toxicity of the first metabolite of alcohol degradation, acetaldehyde (AA) - a toxic and mutagenic metabolite of ethanol [7].

Two major enzyme systems can metabolize alcohol to AA via oxidative degradation ADH (alcohol dehydrogenase) and CYP2E1 of which alcohol dehydrogenase is the system primarily responsible for the processing of lower amounts of alcohol, while CYP2E1 is 
inducible and contributes most to acetaldehyde production during heavy alcohol consumption. Apart from generating AA, CYP2E1 also contributes to oxidative damage by the formation of reactive oxygen species (ROS) such as superoxide anion and hydrogen peroxide [6, 7]. Additionally, the induction of CYP2E1 correlates with NAD phosphate (NADPH) oxidase activity, the generation of hydroxyethyl radicals, lipid peroxidation and the severity of hepatic damage [8]. Toxic aldehydes formed during lipid peroxidation just as acetaldehyde can bind and built-up adducts with lipids, DNA and more. These DNA adducts conduce to mutations in a DNA molecule, damage of nucleotides thus leading to not only toxic but carcinogen results as well [9].

\section{Pathophysiology and progression of ALD}

Central to the progression of alcoholic hepatitis are resident and infiltrating immune cells called macrophages, which have important roles in inducing liver inflammation [4]. Kupffer cells (KCs), the resident macrophages in the liver, reside in the liver sinusoids and provide the first line of defense, serving as potent innate immune cells. KCs can regulate the development of inflammation, depending on their ability to either induce or suppress proinflammatory changes. Because the liver is exposed to countless antigens, pathogens, and toxic substances that come from the intestine via the portal circulation, it must be protected from developing an immune response to such exposure. That's why KCs usually have tolerogenic properties, meaning that they do not respond to all antigens with an immune response. However, excessive alcohol exposure can switch KCs to a proinflammatory phenotype (M1). This type releases multiple proinflammatory cytokines, including TNF $\alpha$, interleukins, and chemokines that attract inflammatory cells from circulation. KCs also are an abundant source of ROS that exacerbate oxidative stress in the liver.

The studies showed that a major factor in the initiation of the inflammatory response by resident macrophages of the liver is endotoxin or lipopolysaccharide (LPS), a cell-wall component of Gram-negative bacteria that translocates from the gut lumen into the portal circulation to reach the liver. Enhanced circulating endotoxin levels in alcoholic hepatitis are caused by alcohol-induced qualitative and quantitative changes in the bacteria that inhabit the gut and increased gut leakiness [10]. In the liver, LPS is sensed by two types of receptors CD14 and toll-like receptor 4 (TLR4) - on the KC surface. These receptors activate KCs and stellate cells to produce reactive oxygen species (ROS) as well as proinflammatory cytokines and chemokines that together with alcohol contribute to hepatocyte damage [11]. Moreover, animal studies have revealed that increased circulating endotoxin levels correlate with the severity of liver disease [12].

Among other prominent factors that can exacerbate liver inflammation are MAA adducts that are produced in alcohol-exposed hepatocytes and are taken up by scavenger receptors on $\mathrm{KCs}$, further promoting the pro-inflammatory response [13].

Other factors contributing to hepatocyte damage include alcohol-induced activation of various immune cells as well as alcohol's effects on the adipose tissue, which results in the production of damage-associated molecular pattern (DAMP) molecules [4]. DAMPs are molecules released by stressed cells undergoing necrosis that act as endogenous danger signals to promote and exacerbate the systemic inflammatory response in the absence of infection $[34,35]$.

\section{Immune dysregulation in ALD}

Exposure to chronic and excessive alcohol consumption, its breakdown metabolites and gut-derived endotoxins dysregulate immune signaling leading to activation of the systemic and local pathways of inflammation $[18,19]$. 
Cytokines are low molecular weight mediators of cellular communication produced by multiple cell types in the liver. Kupffer cells are prominent producers of pro-inflammatory cytokines, such as IL-1 and TNF-, as well as certain anti-inflammatory cytokines, such as IL-10. Hepatocytes are increasingly a recognized source of cytokine production and not just a target of cytokine toxicity in liver injury. Hepatocytes are important producers of chemokines such as IL-8, a major neutrophil chemotactic peptide and an angiogenesis factor and also IL18 , which regulates the inflammatory response.

Abnormal cytokine metabolism is a major feature of ALD. Craig J. McClain et al. [14] were first to observe and report dysregulated TNF- $\alpha$ metabolism in ALD patients with the observation that cultured monocytes (which produce the overwhelming majority of systemic circulating TNF- $\alpha$ and are a surrogate marker for Kupffer cells) from ALD patients spontaneously produced TNF- $\alpha$ and produced significantly more TNF- $\alpha$ in response to an endotoxin (LPS) stimulus than did control monocytes [15] .

Additionally, other several groups reported increased serum concentration levels of TNF- $\alpha$ and TNF- $\alpha$-inducible cytokines/chemokines, such as IL-6, -8 , and -18 , in patients with alcoholic hepatitis and/or cirrhosis, and levels correlated with markers of the acute phase response, liver function, and clinical outcome [15,16,17].

However, insufficient response to corticosteroid treatment among patients with ALD and lack of the positive results in the studies with new therapy options like pentoxifylline, a tumor necrosis factor-alpha (TNF $\alpha$ ) suppressor, or infliximab (an anti-TNF $\alpha$ mouse/human chimeric antibody) force to further research new concepts and pathways of ALD development. The immune-mediated pathophysiological mechanisms of ALD turned out to be far more complex than it was thought and still require further investigation to be fully clarified.

\section{Potencial role of PD1/PDL-1 pathway in pathogenesis of ALD}

Recent studies have elucidated the relevance of the programmed cell death 1 (PD-1) receptor and its ligand PD-L1 in inhibition of self-reactive and inflammatory effector cells and the protection against immune-mediated tissue damage. As a negative regulator, PD-1 exerts a controlling influence on the response of previously activated $\mathrm{T}$ and $\mathrm{B}$ cells by binding its ligands PD-L1/PD-L2.

PD-L1 and PD-L2 are types I transmembrane glycoproteins. The expression of PD-L1 constitutively exists not only on T and B cells, DCs, macrophages, mesenchymal stem cells, and bone marrow-derived mast cells [20] - PD-L1 is also expressed on a large-scale in nonhematopoietic cells such as lung, vascular endothelial, fibroblastic reticular, liver nonparenchymal, and mesenchymal stem cells, pancreatic islets, astrocytes, neurons, and keratinocytes [21, 22]. This wide extent of PDL-1 expression boosts automatically its biological importance in almost every aspect of immune responses. The signals transduced from PD-1 cross-linked with B- or T-cell antigen receptors, restrict the intensity and duration time of the immune response, therefore as a consequence limit immune-induced tissue damage, control resolution of inflammation, and maintain peripheral immune tolerance [23, 24].

The PD-1/PD-L1 pathway has been shown to play an important role in a variety of diseases, including cancer, autoimmune conditions, and chronic infection. It has been already investigated in several experimental animal models as systemic lupus erythematosus, encephalomyelitis, myasthenia gravis, diabetes mellitus, myocarditis, inflammatory bowel diseases, systemic sclerosis and rheumatoid arthritis. The recent studies has showed that its play a critical role in the prevention of disorders caused by impaired immune activation $[25,26]$ and there are the first positive results of various diseases treatment with the use PD1/PDL-1 modulation. However, its impact on the development and progression of ALD 
has not been entirely explained yet. It seems possible that alterations in the checkpoint inhibitor surveillance may be responsible for activation of the inflammatory response in the liver as well as the systemic character of the disease. Therefore, it seems to be justifiable to explore the expression of PD-1 and PD-L1 on peripheral $\mathrm{T}$ and $\mathrm{B}$ lymphocytes, its correlations with the severity of liver dysfunction and conventional markers of inflammation in the course of ALD.

\section{Gender Difference in Alcoholic Liver Disease}

As evidenced by studies, females have higher blood ethanol levels than males after an equivalent dose of alcohol [27] and have a significantly higher relative risk of developing alcohol-related liver disease. According to a study by Eriksson et al. and Sherlock \& Dooley $[28,29]$, chronic alcohol consumption may induce more rapid and more severe liver injury in females than males. Furthermore, females with alcoholic cirrhosis survive a shorter time than males. There are multiple explanations and conceptions for this. First, females are generally smaller than males and also their body water content is smaller than males per kilogram of body weight [30]. Second, alcohol is metabolized with the enzyme gastric ADH, that gastric activity is lower in females than in males. According to Nolen-Hoeksema's study [31], this sex difference in the metabolism of alcohol appears to hold for younger adults but not older adults. ADH activity decreases with age, particularly for males, leading to similar blood alcohol concentrations in older males and females. This forces to further search explanations for different sensibility for toxic effects of ethanol in males and females.

Muñoz-Cruz et al. thoroughly review the growing body of evidence that shows a reciprocal relationship between sex steroids and the immune system. Sex hormones have been suggested to redound in the "female paradox" by binding to receptors present on immune cells, which results in modification of gene expression, lymphocyte proliferation, antigen presentation, and cytokine secretion [32,33]. Females produce higher titers of circulating immunoglobulins, as well as of a variety of autoreactive antibodies. They display a more pronounced humoral immune response against infection in comparison with males [33].

Considering immune dysregulation involvement in ALD progression and dissimilar sensibility to toxic effects of ethanol in males and females, the potential gender-related differences in PD-1/PD-L signaling in ALD development is possible as well.

\section{Conclusions:}

The pathophysiology of ALD is complex and remains not fully researched. It generates a problem in finding the right therapy for patients with alcohol-damaged liver. There are still no pharmacological or nutritional therapies for treating patients with alcoholic liver disease. Alcohol abstinence is an integral part of therapy but liver transplantation remains still the life-saving strategy for patients with end-stage alcoholic liver disease. A better understanding of ALD as a dynamic process and all aspects of immune dysregulation seems to be important for further progress in the development of novel therapies in ALD. Results from a further study on PD1/PDL-1 pathway might be exploited to re-define therapeutic targets and create sex-tailored treatments according to individual patient needs. 


\section{References}

1. World Health Organization (WHO) Global Status Report on Alcohol and Health. Geneva: WHO; 2018. 38-46.

[http://www.who.int/substance_abuse/publications/global_alcohol_report/msb_gsr_2014_1.p df.

2. Tsochatzis EA, Bosch J, Burroughs AK. (2014). Liver cirrhosis. Lancet, 17;383(9930):1749-61.

3. Rehm J, Gmel GE Sr, Gmel G, et al. (2017). The relationship between different dimensions of alcohol use and the burden of disease-an update. Addiction, 112(6):968-1001.

4. Natalia A Osna, Terrence M Donohue Jr, Kusum K Kharbanda. (2017). Alcoholic Liver Disease: Pathogenesis and Current Management. Alcohol Research:current reviews, 38(2): 147-161.

5. Gao B, Battaler R.(2011).Alcoholic Liver Disease: Pathogenesis and New Therapeutic Targets. Gastroenterology, 141(5): 1572-1585.

6. Stickel F, Datz C, Hampe H, Bataller R. Pathophysiology and Management of Alcoholic Liver Disease: Update 2016. Gut and Liver,11(2): 173-188.

7. Konishi M, Ishii H.(2007). Role of microsomal enzymes in development of alcoholic liver diseases. Journal of Gastroenterology and Hepatology, 22(Suppl 1):S7-S10.

8. Bradford BU, Kono H, Isayama F, et al.(2005). Cytochrome P450 CYP2E1, but not nicotinamide adenine dinucleotide phosphate oxidase, is required for ethanol-induced oxidative DNA damage in rodent liver. Hepatology : official journal of the American Association for the Study of Liver Diseases, 41:336-344.

9. Scoccianti C, Cecchini M, Anderson AS, et al.(2015). European Code against Cancer 4th Edition: alcohol drinking and cancer. Cancer Epidemiology, 39(Suppl 1):S67-S74.

10. Bode C, Bode JC. Effect of alcohol consumption on the gut.(2003). Best practice and research. Clinical gastroenterology, 17(4):575-592.

11. Suraweera DB, Weeratunga AN, Hu RW, et al. (2015). Alcoholic hepatitis: The pivotal role of Kupffer cells. World Journal of Gastrointestinal Pathophysiology, 6(4):90-98.

12. Mathurin P, Deng QG, Keshavarzian A, et al. (2000). Exacerbation of alcoholic liver injury by enteral endotoxin in rats. Hepatology : official journal of the American Association for the Study of Liver Diseases, 32(5):1008-1017.

13. Ambade A, Mandrekar P. (2012). Oxidative stress and inflammation: Essential partners in alcoholic liver disease. International Journal of Hepatology, 2012: 853175

14. Craig J McClain, Zhenyuan Song, Shirish S, Barve, et al. 2004). Recent Advances in Alcoholic Liver Disease IV. Dysregulated cytokine metabolism in alcoholic liver disease. AJP Gastrointestinal and Liver Physiology, 287: G497-G502.

15. McClain CJ, Cohen DA. (1989). Increased tumor necrosis factor production by monocytes in alcoholic hepatitis. Hepatology : official journal of the American Association for the Study of Liver Diseases, 9: 349-351.

16. Kamimura $\mathrm{S}$ and Tsukamoto H. (1995). Cytokine gene expression by Kupffer cells in experimental alcoholic liver disease. Hepatology : official journal of the American Association for the Study of Liver Diseases, 21: 1304-1309.

17. Kasztelan-Szczerbinska B, Surdacka A, Slomka M, et al. (2014). Angiogenesis-related biomarkers in patients with alcoholic liver disease: their association with liver disease complications and outcome. Mediators Inflammation, 2014:673032.

18. Gao B, Tsukamoto H. (2016). Inflammation in alcoholic and nonalcoholic fatty liver disease: friend or foe?.Gastroenterology, 150, 1704-1709.

19. Szabo, G, Saha B. (2015). Alcohol's Effect on Host Defense. Alcohol research : current reviews, 37, $159-170$. 
20. Yamazaki T, Akiba H, Iwai H. et al. (2002). Expression of programmed death 1 ligands by murine T cells and APC. Journal of Immunology, 169:5538-45.

21. Agata Y, Kawasaki A, Nishimura H, Ishida Y, et al. (1996). Expression of the PD-1 antigen on the surface of stimulated mouse $\mathrm{T}$ and B lymphocytes. International Immunology, 8:765-72.

22. Keir ME, Butte MJ, Freeman GJ, Sharpe AH. (2008). PD-1 and its ligands in tolerance and immunity. Annual review of immunology, 26:677-704.

23. Boussiotis, VA, Chatterjee P, Li, L. (2014). Biochemical signaling of PD-1 on T cells and its functional implications. The cancer journal. 20, 265-271

24. Bardhan K., Anagnostou T., Boussiotis, V.A. (2012).The PD1:PD-L1/2 Pathway from discovery to clinical implementation. Frontiers of immunology, 7:550.

25. Zamani, MR, Aslani S, Salmaninejad A, et al. (2016). PD-1/PD-L and autoimmunity: A growing relationship. Cellular immunology, 310, 27-41.

26. Dinesh, RK, Hahn BH, Singh RP.(2010). PD-1, gender, and autoimmunity. Autoimmunity Reviews, 9, 583-587.

27. Shimizu I, Yoshikawa H, Kamochi M, Nakayama Y. (2012). Gender difference in alcoholic liver disease. INTECH Open Access Publisher, 11:24-40.

28. Eriksson CJ, Fukunaga T, Sarkola T, Lindholm H \& Ahola L. (1996). Estrogenrelatedacetaldehyde elevation in women during alcohol intoxication. Alcoholism, clinical and experimental research, 20:1192-1195.

29. Sherlock S \& Dooley J.(2002). Diseases of the liver and biliary system. Blackwell Science,Oxford,494.

30. Frezza M, di PC, Pozzato G, Terpin M, Baraona E \& Lieber CS. (1990). High blood alcohol levels in women. The role of decreased gastric alcohol dehydrogenase activity and first-pass metabolism. The New England journal of medicine, 322:95-99.

31. Nolen-Hoeksema S. (2004). Gender differences in risk factors and consequences for alcohol use and problems. Clinical psychology review, 24:981-1010.

32. Whitacre CC. (2001).Sex differences in autoimmune disease. Nature immunology, 2, 777-780

33. Muñoz-Cruz S, Togno-Pierce C, Morales-Montor J. (2011). Non-reproductive effects of sex steroids: their immunoregulatory role. Current topics in medicinal chemistry, 11(13):1714-1727

34. Jong Seong Roh, Dong Hyun Sohn. (2018). Damage-Associated Molecular Patterns in Inflammatory Diseases. Immune Network, 18(4): e27

35. Mihm S. (2018). Danger-Associated Molecular Patterns (DAMPs): Molecular Triggers for Sterile Inflammation in the Liver. International journal of molecular sciences, 19(10):3104 